\title{
Quantum Communications and Quantum Computation
}

\begin{abstract}
Quantum entanglement, the real-time synchronization between two coupled quanta distributed afar, has intrigued physicists for a long time as a "spooky action" described by Albert Einstein. How the quanta of the coherent pair "interact" with each other despite the long distance remains a mystery, but the theoretically perfect security promised by the inherent physics encourages scientists to explore possible ways to utilize it for information encryption After all, a message sealed by quantum cryptography would be totally immune to hackers.

Represented by the University of Science and Technology of China (USTC), various institutions under the Chinese Academy of Sciences (CAS) have been continuously working for over a decade to solve fundamental issues in quantum mechanics, and has developed a series of expandable technologies for quantum information processing, which have found their applications in quantum communications and quantum computation.
\end{abstract}

\section{Quantum Encryption}

Theoretically, in a quantum cryptography system no eavesdropping is possible. According to the principle of quantum entanglement, any effort to intercept or copy the secret key being sent from one to another of an entangled pair, would change the state of the system. and hence would be detected immediately from either the sender or the receiver terminal.

Looking at the promising prospects, the USTC team started early in scientific explorations into quantum physics, and also the R\&D of quantum communication technologies, aimed at establishing high-speed, longdistance quantum communication networks. Supported by CAS, the team has assumed a series of major projects. and has made great progress in quantum physics. particularly successful preparation and distribution of entangled photon pairs across different distances.

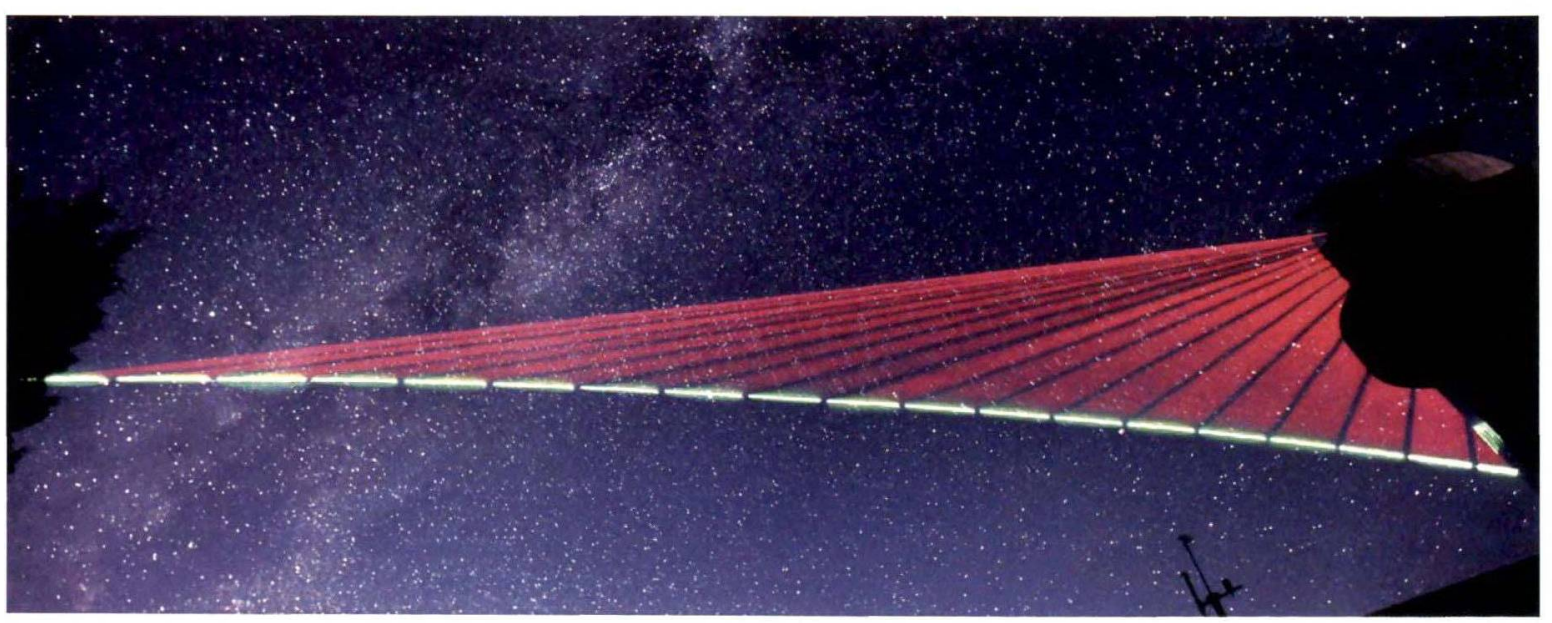




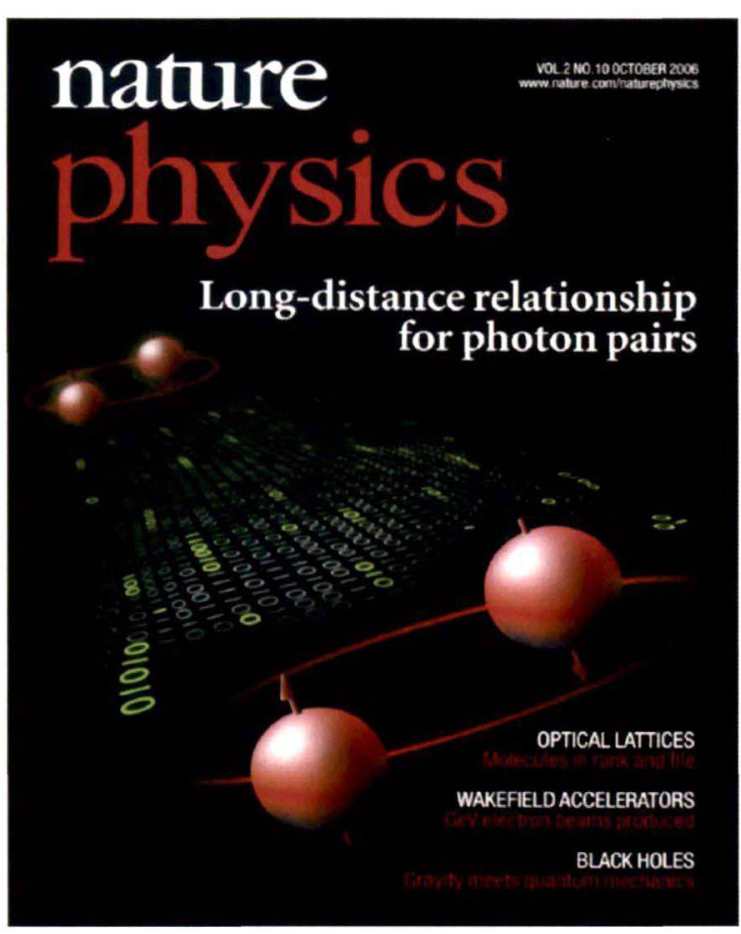

Early start: A team at USTC articulates how they achieved teleportation of a complex quantum system in Nature Physics in 2006. (Credit: USTC)

Drawing on their buildup in quantum physics, the team succeeded in building the first general quantum communication network in Hefei, the capital city of east China's Anhui Province, as early as in 2008. In 2009, the team took the lead in extending the distance of decoy state quantum communications to $200 \mathrm{~km}$. In 2012 , the team completed building a large-scale (composed of a total of 46 nodes) quantum communication network covering the whole city of Hefei, the first of this kind in the world. This marked the maturity of technologies for large-capacity quantum communication network in metropolitan areas.

However, to establish a network for quantum communications for real application, human beings have to break through a bottleneck: due to the loss induced by collisions between the photon pairs and the gas molecules in the atmosphere, the entanglement cannot survive a very long distance. Integrating satellites into the network might solve this problem. given that transecting the space between the ground and a satellite orbiting the Earth could be much easier than traveling through the free space between two cities situated $100 \mathrm{~km}$ apart. The densely compact atmosphere near to the ground would destruct the long-distance relationship between the pair: while the much thinner air high above the ground would be a haven for the couple. The farther from the ground, the thinner the atmosphere; at an altitude over $500 \mathrm{~km}$ above the Earth, the chances of collisions, and hence loss, could be greatly reduced.

Therefore, using satellites for relay transmission, a global network of quantum communications could be established.

To explore the possibility of quantum communications via satellites, the team made intensive efforts in 2012 and 2013 to realize quantum teleportation and entanglement distribution across a free space of over $100 \mathrm{~km}$ distance, and verified the feasibility of satellite-earth quantum communication with comprehensive ground experiments. Eventually in August 2016, they saw the launching of their brainchild, the first satellite for quantum science experiment in space - Micus, which is named after Mozi (Mo-tse), a philosopher of ancient China who explored the pin-hole imaging phenomenon as early as around 2500 years ago.

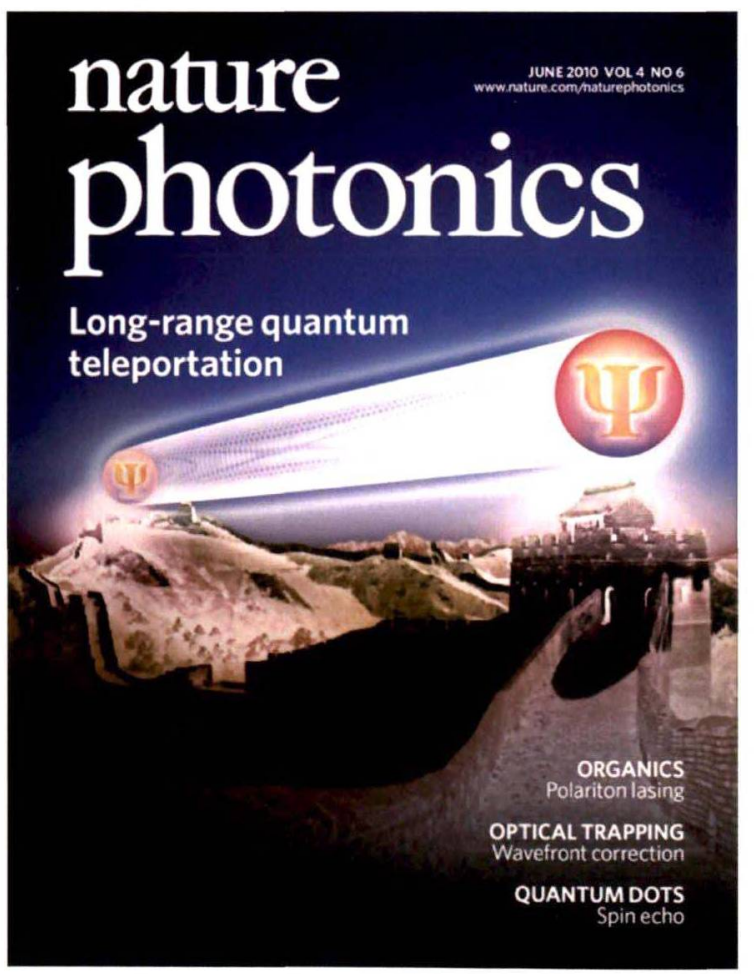

The USTC team reports in Nature Photonics the successful quantum teleportation across a $16 \mathrm{~km}$-long distance in 2010 , marking a milestone in long-range transmission of quantum information. (Credit: USTC) 


\section{Quantum Communications Extending to Outer Space}

The launching of Micius has further boosted the advancement in this field by demonstrating the feasibility of building a global communication network based on ground stations and satellites. Led by Prof. PAN Jianwei, a CAS Member from USTC, integrated forces from a series of institutions under CAS tackled a series of technological barriers and blazed the trail to a network for quantum communications across continents.

By August 2017, Micius had reported success in all three preset space experiments - the bidirectional satellite-ground distribution of quantum entanglement, encrypted quantum keys, and quantum teleportation - all to and from the Satellite over a distance of $1,000^{+}$ $\mathrm{km}$. Around the same time, an experiment aboard Tiangong-2, the space lab of China, succeeded in distributing quantum encrypted keys between the ground station and the spacecraft.

September of the same year witnessed the opening of a trunk line for quantum communications spreading from Beijing to Shanghai, connecting multiple cities in China. This marks the first ever of such efforts in the world. Based on this trunk line and the ground- satellite links, the team made the first quantum video call from China to Europe, hailing a network for widearea quantum communication integrating space- and ground-based facilities.

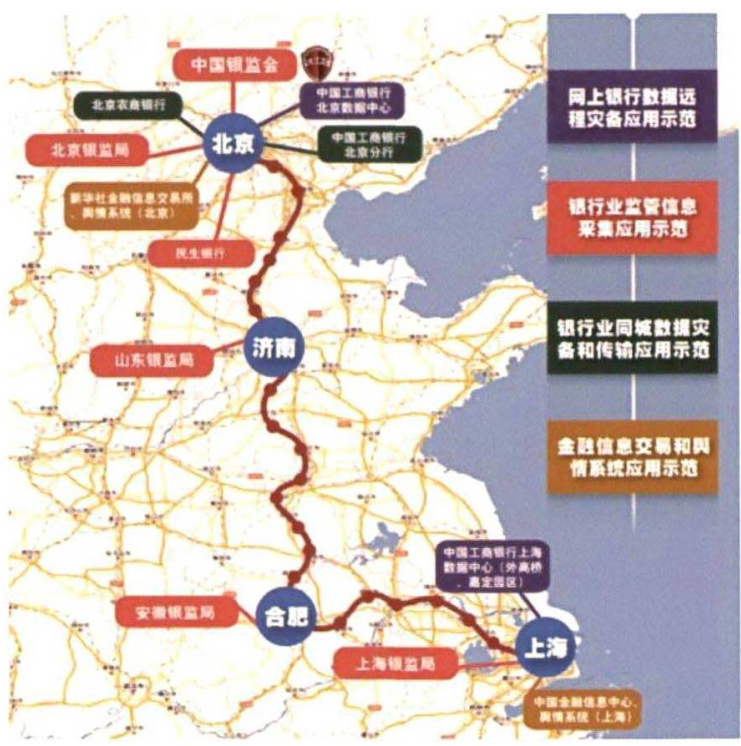

The arterial line for quantum communications between Beijing and Shanghai. (Credit: USTC)

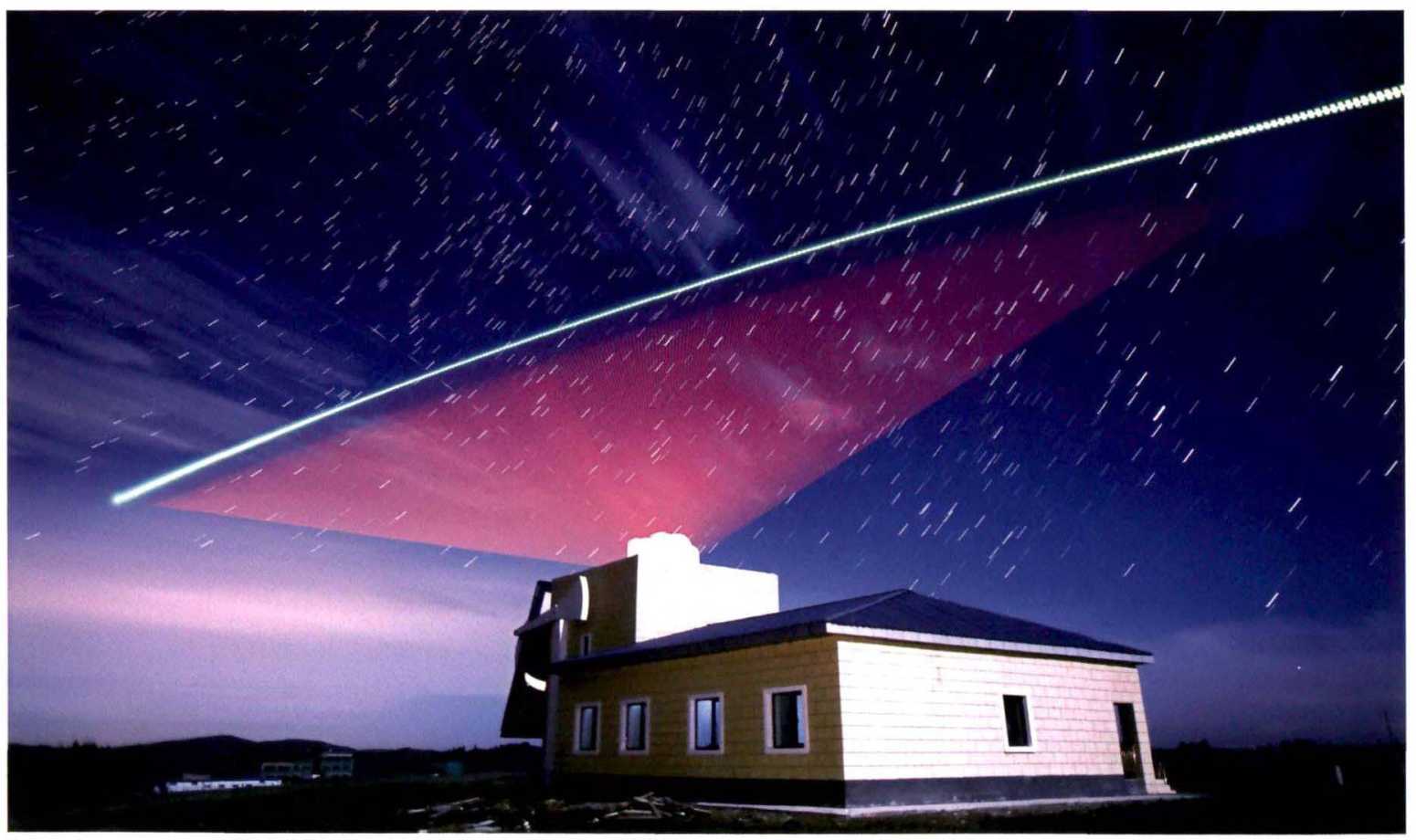

A time-lapse exposure (10 to 15 seconds) image taken by the USTC team featuring the satellite Micius passing over the Nanshan optical ground station (OGS). The green line is the bacon laser from the satellite to the ground, and the red light the bacon laser from the ground station to the satellite. (Photo by ZHU Jin) 


\section{Quantum Computation}

The USTC team is also a leading force concerning the preparation and manipulation of multi-particle quantum entanglement, the physics basis for quantum computation. Since 2004, the team has maintained the position of world record setter and keeper in terms of the number of entangled photons achieved in a system.

In 2015. the team took the lead to achieve quantum teleportation of multiple degrees of freedom, a feat later evaluated by the Institute of Physics of UK as a "Breakthrough of the Year". Later in January 2016. PAN's team won the First Prize from the National S\&.T Awards for Natural Sciences, due to their systematic research in multiple-photon entanglement and interference measurement.

In December 2016, the team further succeeded in achieving ten-photon entanglement, once again updating the world record maintained by themselves.

May 2017 witnessed the successful development of the first prototype quantum computer based on

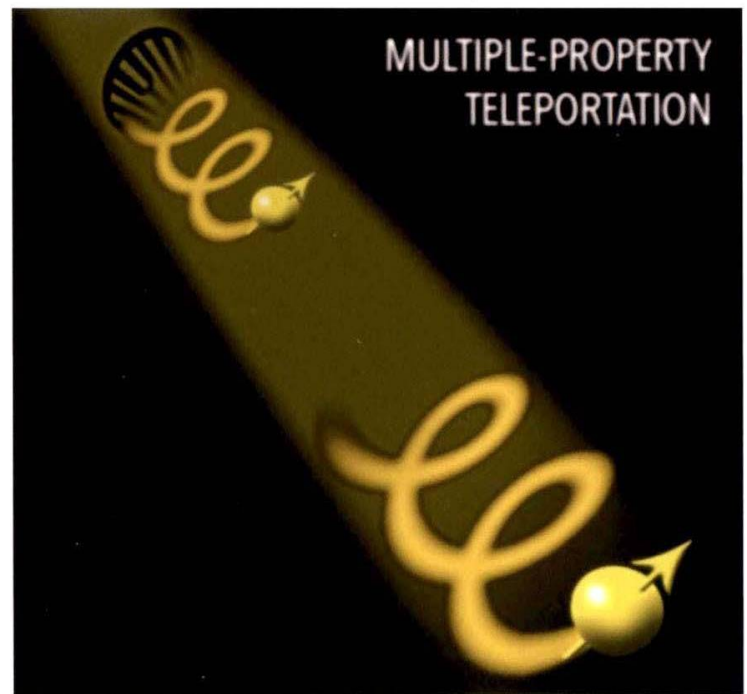

The team reports successful quantum teleportation of multiple degrees of freedom of a single photon in Nature in 2015. (Credit: USTC) single photon. in which the quantum entanglement between ten superconductive quantum bits (qubits) was achieved. This feat was voted into the list of leading achievements at the Fourth World Internet Conference. In February the next year, a platform for quantum computation developed in cooperation with Alibaba Cloud went online, becoming the second system in the world offering quantum computation cloud service of over 10 qubits, ensuing a system developed by IBM.

The team went on to achieve 18 -qubit quantum entanglement in July 2018, further raising the bar for a world record, as well as our expectation for future quantum computation.

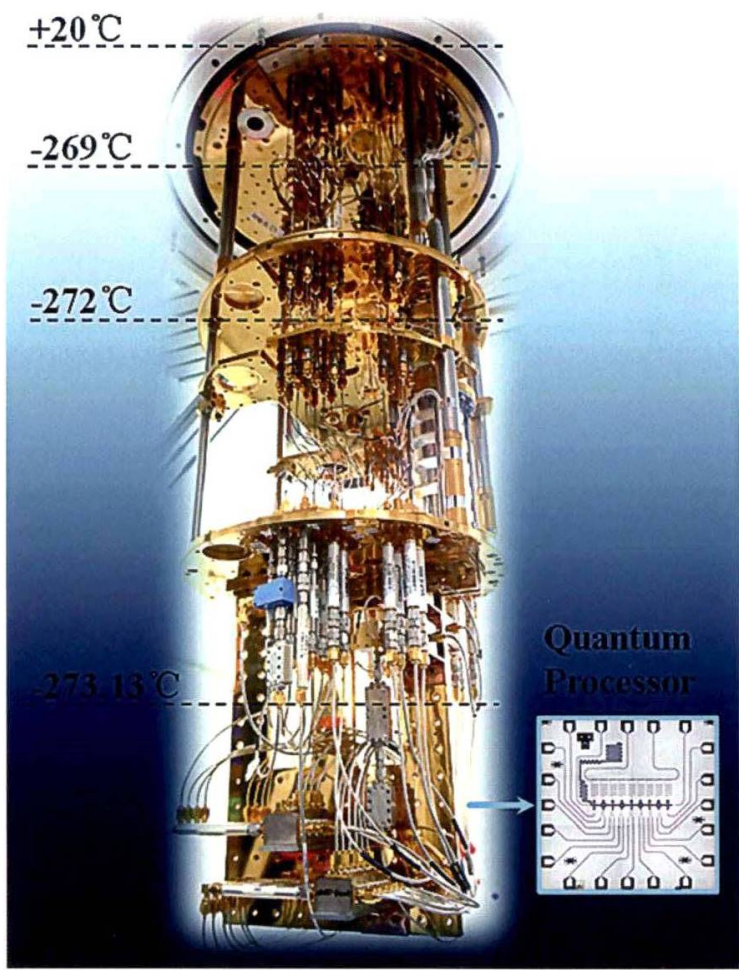

Concept of photon computer . (Credit: USTC) 